\title{
Use of Vaccinia Virus Smallpox Vaccine in Laboratory and Health Care Personnel at Risk for Occupational Exposure to Orthopoxviruses - Recommendations of the Advisory Committee on Immunization Practices (ACIP), 2015
}

\author{
Brett W. Petersen, $\mathrm{MD}^{1}$; Tiara J. Harms, MS, $\mathrm{MPH}^{2}$; Mary G. Reynolds, $\mathrm{PhD}^{1}$; Lee H. Harrison, $\mathrm{MD}^{3,4}$
}

On June 25, 2015, the Advisory Committee on Immunization Practices (ACIP) recommended routine vaccination with live smallpox (vaccinia) vaccine (ACAM2000) for laboratory personnel who directly handle 1) cultures or 2) animals contaminated or infected with replication-competent vaccinia virus, recombinant vaccinia viruses derived from replicationcompetent vaccinia strains (i.e., those that are capable of causing clinical infection and producing infectious virus in humans), or other orthopoxviruses that infect humans (e.g., monkeypox, cowpox, and variola) (recommendation category: A, evidence type 2 [Box]). Health care personnel (e.g., physicians and nurses) who currently treat or anticipate treating patients with vaccinia virus infections and whose contact with replication-competent vaccinia viruses is limited to contaminated materials (e.g., dressings) and persons administering ACAM2000 smallpox vaccine who adhere to appropriate infection prevention measures can be offered vaccination with ACAM2000 (recommendation category: B, evidence type 2 $[\mathrm{Box}])$. These revised recommendations update the previous ACIP recommendations for nonemergency use of vaccinia virus

Recommendations for routine use of vaccines in children, adolescents, and adults are developed by the Advisory Committee on Immunization Practices (ACIP). ACIP is chartered as a federal advisory committee to provide expert external advice and guidance to the Director of the Centers for Disease Control and Prevention (CDC) on use of vaccines and related agents for the control of vaccine-preventable diseases in the civilian population of the United States. Recommendations for routine use of vaccines in children and adolescents are harmonized to the greatest extent possible with recommendations made by the American Academy of Pediatrics (AAP), the American Academy of Family Physicians (AAFP), and the American College of Obstetricians and Gynecologists (ACOG). Recommendations for routine use of vaccines in adults are harmonized with recommendations of AAFP, ACOG, the American College of Physicians (ACP), and the American College of Nurse-Midwives (ACNM). ACIP recommendations adopted by the CDC Director become agency guidelines on the date published in the Morbidity and Mortality Weekly Report (MMWR). Additional information regarding ACIP is available at http://www.cdc.gov/vaccines/acip. smallpox vaccine for laboratory and health care personnel at risk for occupational exposure to orthopoxviruses (1). Since 2001, when the previous ACIP recommendations were developed, ACAM2000 has replaced Dryvax as the only smallpox vaccine licensed by the U.S. Food and Drug Administration (FDA) and available for use in the United States (2). These recommendations contain information on ACAM2000 and its use in laboratory and health care personnel at risk for occupational exposure to orthopoxviruses.

\section{Background}

Smallpox vaccines containing vaccinia virus were used to successfully eradicate smallpox as a disease of humans (3). Eradication was made possible by the ability of vaccinia virus to induce cross-protective immunity against other viruses within the orthopoxvirus genus capable of producing human infection (e.g., variola, monkeypox, and cowpox) (3). ACAM2000 (Smallpox [Vaccinia] Vaccine, Live) is currently the only smallpox vaccine licensed by FDA and available for use in the United States. The license for Dryvax vaccine, the smallpox vaccine previously recommended by ACIP, was withdrawn in

BOX. The U.S. Advisory Committee on Immunization Practices system for grading evidence and recommendations*

\section{Recommendation categories}

Category A: Recommendation that applies to all persons in an age- or risk-based group.

Category B: Recommendation for individual clinical decision-making.

\section{Type or quality of evidence}

1. Randomized controlled trials (RCTs), or overwhelming evidence from observational studies.

2. RCTs with important limitations, or exceptionally strong evidence from observational studies.

3. RCTs with notable limitations, or observational studies.

4. RCTs with several major limitations, observational studies with important limitations, or clinical experience and observations.

\footnotetext{
*Adopted from the GRADE (Grading of Recommendations Assessment, Development, and Evaluation) system.
} 
2008, and all remaining supplies of this vaccine were subsequently destroyed (2).

ACAM2000 is a vaccinia virus vaccine derived from a plaque-purified clone of the same New York City Board of Health strain that was used to manufacture Dryvax vaccine. ACAM2000 is grown in African green monkey kidney (Vero) cells and tested to be free of known adventitious agents (4). Safety data from ACAM2000 clinical trials indicate a similar safety profile to Dryvax, including a risk for serious adverse events (e.g., progressive vaccinia, postvaccinial encephalitis, and eczema vaccinatum) $(5,6)$. Myopericarditis has also been associated with ACAM2000 and is estimated to occur at a rate of 5.7 per 1,000 primary vaccinees based on clinical trial data (G).

ACAM2000 is provided as a lyophilized preparation of purified live virus containing the following nonactive excipients: $6 \mathrm{mM}-8 \mathrm{mM}$ HEPES (pH 6.5-7.5), 2\% human serum albumin United States Pharmacopeia (USP), 0.5\%-0.7\% sodium chloride USP, 5\% mannitol USP, and trace amounts of neomycin and polymyxin B (6). Diluent for ACAM2000 contains $50 \%(\mathrm{v} / \mathrm{v})$ glycerin USP and $0.25 \%(\mathrm{v} / \mathrm{v})$ phenol USP in water for injection USP. Diluent is supplied as $0.6 \mathrm{~mL}$ of liquid in $3 \mathrm{~mL}$ clear glass vials (6).

ACAM2000 is administered in a single dose by the percutaneous route (scarification) using 15 jabs of a stainless steel bifurcated needle that has been dipped into the reconstituted vaccine (6). Following successful administration of vaccine, ACAM2000 produces vaccination site lesions containing infectious vaccinia virus capable of transmission through autoinoculation and inadvertent inoculation of close contacts of vaccinees. The development of vaccination site lesions may be modified or greatly reduced in revaccinees $(3,6)$.

Poxviruses are increasingly being used in biomedical research for a wide range of purposes. Vaccinia virus is the most frequently studied poxvirus and serves as the prototype of the orthopoxvirus genus. It has not only been used in the area of basic virology but also as both an immunology tool and potential vaccine vector because of its ability to serve as a vector for the expression of foreign genes (antigens) $(7,8)$. Many strains of vaccinia virus exist with different levels of virulence in humans and animals. Distinguishing between replicationcompetent and replication-deficient poxvirus strains is useful in establishing the risk they pose to persons who might be occupationally exposed to such viruses. Replication-deficient poxvirus strains can be defined as those that do not produce infectious virus in humans (and therefore do not cause clinical infection), and as such, pose a substantially lower risk compared with replication-competent poxvirus strains, which are capable of causing clinical infection in humans as well as producing infectious virus that can be transmitted to others. Modified vaccinia Ankara (MVA), NYVAC, TROVAC, and
ALVAC are examples of replication-deficient poxvirus strains $(9,10)$. The categories replication-competent and replicationdeficient replace the previous poxvirus strain categories of highly attenuated and nonhighly attenuated to add clarity and specificity to the vaccination recommendations (1). Persons at risk for occupational exposure to orthopoxviruses might include laboratory personnel who have contact or work with live orthopoxviruses or clinical samples from suspected cases of orthopoxvirus infection, animal care personnel who have direct contact with orthopoxvirus-inoculated or -infected animals or their secretions, and health care personnel (e.g., physicians and nurses) involved in caring for orthopoxvirus-infected persons or administering biological agents containing orthopoxviruses.

\section{Methods}

These recommendations were developed using the Grading of Recommendations Assessment, Development and Evaluation (GRADE) methodology (Box) (11-13). GRADE steps include defining specific questions, identifying important health outcomes, summarizing evidence for important outcomes, assessing quality of evidence, and formulating recommendations. Principal considerations for formulating recommendations include balance of benefits and harms; quality of evidence; values and preferences; and health economic analyses. The central policy question for this policy note was whether routine vaccination with ACAM2000 should be recommended for laboratory and health care personnel at risk for occupational exposure to orthopoxviruses (13).

\section{Rationale and Evidence}

ACIP considered the risk for infection, the risk for an adverse event following vaccination, and the benefit from vaccination in developing these recommendations. Vaccinia virus smallpox vaccine has been recommended by ACIP for the protection of laboratory personnel against orthopoxviruses since 1980 . However, 14 orthopoxvirus infections were reported in laboratory personnel in the United States during 2004-2014; 13 of these infections occurred in laboratory personnel who were not vaccinated according to ACIP recommendations (8) (CDC unpublished data 1/1/2015). Although these data indicate the presence of risk, it is difficult to quantify the absolute number of persons at risk for occupational exposure to orthopoxviruses because the size of the population at risk is not known and vaccinia virus and cowpox virus exposures and infections among this population are not notifiable events. During the same 2004-2014 period, no reports of preventable vaccine-associated serious adverse events (e.g., eczema vaccinatum, progressive vaccinia, or contact transmission) were documented among laboratory and health care personnel at risk for occupational exposure who had been vaccinated with 
smallpox vaccine. Furthermore, data from U.S. military personnel and civilian first responders vaccinated during smallpox vaccination campaigns that were initiated in 2002 indicate that the incidence of serious adverse events overall was lower than previously reported in 1968 (14-16). Although serious adverse events have occurred, this decrease in incidence is likely attributable to more stringent prevaccination screening procedures to identify persons who should not receive the vaccine, to increased use of protective bandages to cover the vaccination site, and to enhanced education of vaccinees compared with the routine vaccination practices in place in the 1960s. Vaccination with ACAM2000 is expected to provide benefit to persons at risk for occupational exposure to orthopoxviruses, given the ability of vaccinia virus smallpox vaccines to induce cross-protective immunity against other viruses within the orthopoxvirus genus.

\section{Recommendations}

Laboratory and health care personnel at risk for occupational exposure to orthopoxviruses should follow recommended biosafety guidelines and adhere to published infection prevention and control procedures (17-19). Laboratories using both replication-competent and replication-deficient vaccinia virus strains where working areas for these viruses cannot be clearly segregated should follow increased biosafety precautions because laboratory infections caused by contamination have been previously documented $(8,17)$. Persons with immunocompromising conditions or other contraindications to vaccination are at increased risk for severe disease if an occupational exposure occurs.

Routine vaccination with ACAM2000 is recommended for laboratory personnel who directly handle 1) cultures or 2) animals contaminated or infected with replication-competent vaccinia virus, recombinant vaccinia viruses derived from replication-competent vaccinia strains (i.e., those that are capable of causing clinical infection and producing infectious virus in humans), or other orthopoxviruses that infect humans (e.g., monkeypox, cowpox, and variola) (recommendation category: A, evidence type 2 [Box]). However, vaccination with ACAM2000 is not recommended for persons who work only with replication-deficient poxvirus strains (e.g., MVA,
NYVAC, TROVAC, and ALVAC) (recommendation category: A, evidence type 2 [Box]).

Laboratory personnel working with replication-competent vaccinia viruses and recombinant viruses developed from replication-competent vaccinia viruses should be revaccinated with ACAM2000 at least every 10 years (recommendation category: A, evidence type 2 [Box]). To ensure an increased level of protection against more virulent orthopoxviruses (e.g., variola, monkeypox), revaccination with ACAM2000 every 3 years is recommended for personnel handling these viruses (recommendation category: A, evidence type 2 [Box]) (Table 1). Public health and health care volunteers who were vaccinated as responders in the U.S. Civilian Smallpox Preparedness and Response Program should refer to the October 2008 CDC Interim Guidance for Revaccination of Eligible Persons who Participated in the US Civilian Smallpox Preparedness and Response Program (http://emergency.cdc.gov/agent/smallpox/ revaxmemo.asp).

Health care personnel (e.g., physicians and nurses) or animal care personnel whose contact with replication-competent vaccinia viruses is limited to contaminated materials (e.g., dressings or cages), but who adhere to appropriate infection prevention measures, are at lower risk for inadvertent infection than laboratory personnel. Similarly, persons administering ACAM2000 smallpox vaccine to laboratory and health care personnel at risk for occupational exposure to orthopoxviruses can decrease the risk for inadvertent infection through recommended infection prevention measures. However, because of a theoretical risk for infection, vaccination with ACAM2000 can be offered to health care or animal care personnel, provided individual persons have no specified contraindications to vaccination (recommendation category: $\mathrm{B}$, evidence type 2 [Box]). Persons with an orthopoxvirus exposure should be evaluated by a health care provider and clinical management decisions, including postexposure smallpox vaccination should be made on a case-by-case basis in consultation with public health authorities.

\section{Precautions and Contraindications}

Nonemergency use of ACAM2000 should be avoided in persons with increased risk for adverse events following

TABLE 1. Recommendations for revaccination of laboratory and health care personnel at risk for occupational exposure to orthopoxviruses

\begin{tabular}{ll}
\hline Orthopoxvirus & Revaccination schedule \\
\hline Replication-competent vaccinia viruses and recombinant viruses developed from replication-competent vaccinia viruses & At least every 10 years \\
More virulent orthopoxviruses (e.g., variola, monkeypox) & Every 3 years \\
Replication-deficient vaccinia viruses and recombinant viruses developed from replication-deficient vaccinia viruses* & Not recommended \\
\hline
\end{tabular}

* Laboratories that use both replication-competent and replication-deficient vaccinia virus strains but where working areas for these viruses cannot be clearly segregated should follow increased biosafety precautions because laboratory infections due to contamination have previously been documented. Sources: MacNeil A, Reynolds MG, Damon IK. Risks associated with vaccinia virus in the laboratory. Virology 2009;385:1-4; Chosewood LC, Wilson DE. CDC; National Institutes of Health. Biosafety in microbiological and biomedical laboratories. 5th ed. Washington, DC: US Department of Health and Human Services, Public Health Service, CDC, National Institutes of Health; 2009. 
administration of smallpox vaccine. Contraindications for nonemergency use of ACAM2000 include persons with a history or presence of atopic dermatitis, persons with other active exfoliative skin conditions (e.g., eczema, burns, impetigo, varicella zoster virus infection, herpes simplex virus infection, severe acne, severe diaper dermatitis with extensive areas of denuded skin, psoriasis, or Darier disease [keratosis follicularis]); persons with conditions associated with immunosuppression (e.g., human immunodeficiency virus [HIV] infection or acquired immune deficiency syndrome [AIDS], leukemia, lymphoma, generalized malignancy, solid organ transplantation, or therapy with alkylating agents, antimetabolites, radiation, tumor necrosis factor [TNF] inhibitors, or highdose corticosteroids [ $\geq 2 \mathrm{mg} / \mathrm{kg}$ body weight or $\geq 20 \mathrm{mg} /$ day of prednisone or its equivalent for $\geq 2$ weeks], hematopoietic stem cell transplant recipients $<24$ months post-transplant or $\geq 24$ months, but who have graft-versus-host disease or disease relapse, or autoimmune disease [e.g. systemic lupus erythematosus] with immunodeficiency as a clinical component); persons aged $<1$ year; women who are pregnant or breastfeeding; persons with a serious allergy to any component of ACAM2000; persons with known underlying heart disease with or without symptoms (e.g., coronary artery disease or cardiomyopathy); and primary vaccinees with three or more known major cardiac risk factors (i.e., hypertension, diabetes, hypercholesterolemia, heart disease at age 50 years in a firstdegree relative, and smoking) (recommendation category: A, evidence type 2). Data from clinical trials and epidemiologic studies suggest that primary vaccinees might be at increased risk for myopericarditis $(20,21)$. Although the specific risk factors for myopericarditis following smallpox vaccination have not been identified, the consequences of myopericarditis are more likely to be severe in persons with known heart disease or cardiac risk factors than in persons without these conditions.

Given the risk for vaccinia virus transmission from recently vaccinated persons through inadvertent inoculation, nonemergency use of ACAM2000 is also contraindicated in persons with household contacts with a history or presence of atopic dermatitis, other active exfoliative skin conditions (e.g., eczema, burns, impetigo, varicella zoster, herpes, severe acne, severe diaper dermatitis with extensive areas of denuded skin, psoriasis, or Darier disease [keratosis follicularis]); conditions associated with immunosuppression (e.g., HIV/AIDS, leukemia, lymphoma, generalized malignancy, solid organ transplantation, or therapy with alkylating agents, antimetabolites, radiation, TNF inhibitors, or high-dose corticosteroids [i.e., $\geq 2 \mathrm{mg} / \mathrm{kg}$ body weight or $20 \mathrm{mg} /$ day of prednisone or its equivalent for $\geq 2$ weeks], hematopoietic stem cell transplant recipients $<24$ months post-transplant or $\geq 24$ months, but who have graft-versus-host disease or disease relapse, or autoimmune disease [e.g. systemic lupus erythematosus] with immunodeficiency as a clinical component); household contacts aged $<1$ year; and household contacts who are pregnant (recommendation category: A, evidence type 2 [Box]). Household contacts include persons with prolonged intimate contact with the potential vaccinee (e.g. sexual contacts) and others who might have direct contact with the vaccination site or with potentially contaminated materials (e.g., dressings or clothing) (Table 2). ACIP also does not recommend nonemergency vaccination with ACAM2000 for children and adolescents aged $<18$ years.

TABLE 2. Contraindications to using ACAM2000 smallpox vaccine in laboratory and health care personnel at risk for occupational exposure to orthopoxviruses

\begin{tabular}{|c|c|c|c|}
\hline Contraindication & Primary vaccinees & Revaccinees & Household contacts* \\
\hline History or presence of atopic dermatitis & $\sqrt{ }$ & $\sqrt{ }$ & $\sqrt{ }$ \\
\hline Other active exfoliative skin conditions ${ }^{\dagger}$ & $\sqrt{ }$ & $\sqrt{ }$ & $\sqrt{ }$ \\
\hline Conditions associated with immunosuppression ${ }^{\S}$ & $\sqrt{ }$ & $\sqrt{ }$ & $\sqrt{ }$ \\
\hline Pregnancy & $\sqrt{ }$ & $\sqrt{ }$ & $\sqrt{ }$ \\
\hline Aged $<1 \mathrm{yr}^{\natural}$ & $\sqrt{ }$ & $\sqrt{ }$ & $\sqrt{ }$ \\
\hline Breastfeeding & $\sqrt{ }$ & $\sqrt{ }$ & \\
\hline Serious vaccine component allergy & $\sqrt{ }$ & $\sqrt{ }$ & \\
\hline Known underlying heart disease (e.g., coronary artery disease or cardiomyopathy) & $\sqrt{ }$ & $\sqrt{ }$ & \\
\hline Three or more known major cardiac risk factors ${ }^{* *}$ & $\sqrt{ }$ & & \\
\hline
\end{tabular}

* Household contacts include persons with prolonged intimate contact with the potential vaccinee (e.g., sexual contacts) and others who might have direct contact with the vaccination site or with potentially contaminated materials (e.g., dressings or clothing).

+ Conditions include eczema, burns, impetigo, varicella zoster, herpes, severe acne, severe diaper dermatitis with extensive areas of denuded skin, psoriasis, or Darier disease (keratosis follicularis).

$\S$ Conditions include human immunodeficiency virus/acquired immune deficiency syndrome infection, leukemia, lymphoma, generalized malignancy, solid organ transplantation, therapy with alkylating agents, antimetabolites, radiation, tumor necrosis factor inhibitors, high-dose corticosteroids, being a recipient with hematopoietic stem cell transplant $<24$ months post-transplant or $\geq 24$ months but with graft-versus-host disease or disease relapse, or having autoimmune disease with immunodeficiency as a clinical component.

" Vaccination of infants aged $<1$ year is contraindicated. Additionally, the Advisory Committee on Immunization Practices does not recommend vaccinating children and adolescents aged $<18$ years.

** Major cardiac risk factors include hypertension, diabetes, hypercholesterolemia, heart disease at age 50 years in a first-degree relative, and smoking. 


\section{Summary}

What is currently recommended?

In 2001, the Advisory Committee on Immunization Practices approved revised recommendations that laboratory and health care personnel occupationally exposed to vaccinia virus, recombinant vaccinia viruses, and other orthopoxviruses that can infect humans be vaccinated with Dryvax smallpox vaccine.

Why are the recommendations being modified now?

In 2007, ACAM2000 was licensed by the U.S. Food and Drug Administration and replaced Dryvax as the only smallpox vaccine available for use in the United States. The evidence supporting routine vaccination with ACAM2000 for laboratory personnel at risk for occupational exposure to orthopoxviruses was evaluated using the Grading of Recommendations, Assessment, Development, and Evaluation framework and determined to be type 2 (moderate level of evidence); the recommendation was designated as a Category A recommendation.

What are the new recommendations?

Routine vaccination with ACAM2000 is recommended for laboratory personnel who directly handle 1) cultures or 2) animals contaminated or infected with replicationcompetent vaccinia virus, recombinant vaccinia viruses derived from replication-competent vaccinia strains (i.e., those that are capable of causing clinical infection and producing infectious virus in humans), or other orthopoxviruses that infect humans (e.g., monkeypox, cowpox, and variola) (recommendation category: A, evidence type 2). Health care personnel (e.g., physicians and nurses) who currently treat or anticipate treating patients with vaccinia virus infections and whose contact with replication-competent vaccinia viruses is limited to contaminated materials (e.g., dressings) and persons administering ACAM2000 smallpox vaccine who adhere to appropriate infection prevention measures can be offered vaccination with ACAM2000 (recommendation category: B, evidence type 2).

Persons with inflammatory eye disease might be at increased risk for inadvertent inoculation as a result of touching or rubbing the eye. Therefore, deferring vaccination is prudent for persons with inflammatory eye diseases requiring steroid treatment until the condition resolves and the course of therapy is complete (recommendation category: $\mathrm{B}$, evidence type 4 [Box]).

Adverse events occurring after administration of any vaccine should be reported to the Vaccine Adverse Event Reporting System (VAERS). Reports can be submitted to VAERS online, by fax, or by mail. Additional information about VAERS is available by telephone (1-800-822-7967) or online (https:// vaers.hhs.gov).

\section{ACAM2000 Availability}

CDC is the only source of ACAM2000 for civilians. CDC will provide ACAM2000 to protect laboratory and other health care and animal care personnel whose occupations place them at risk for exposure to vaccinia and other orthopoxviruses, including recombinant vaccinia viruses. Vaccine should be administered under the supervision of a physician selected by the requesting institution. Vaccine will be shipped to the responsible physician. Requests for vaccine, including the reason for the request, should be referred to the following: CDC Drug Service, Division of Scientific Resources, National Center for Emerging and Zoonotic Infectious Diseases, Office of Infectious Diseases,Mailstop D-09, Atlanta, GA 30329; telephone: 404-639-3670; fax: 404-639-3717; e-mail: drugservice@cdc.gov.

\section{Future Directions}

ACIP will review these recommendations as new information or developments related to orthopoxvirus disease, smallpox vaccines (including licensure of additional smallpox vaccines), smallpox vaccine adverse events, and the experience gained in the implementation of these recommendations becomes available. Revised recommendations will be developed as needed.

\section{Acknowledgments}

Members of the Advisory Committee on Immunization Practices (ACIP); members of the ACIP Smallpox Vaccine Workgroup (ACIP member roster for July 2014-June 2015 is available online [http:// www.cdc.gov/vaccines/acip/committee/members.html]); Faruque Ahmed, Nancy M. Bennett, Maria Cano, Mark D. Challberg, Paul Chaplin, Emily A. Cloessner, Limone C. Collins, Inger K. Damon, Michael D. Decker, Renata J. Engler, Doran L. Fink, Jesse R. Geibe, Richard L. Gorman, Richard N. Greenberg, Laura Hughes-Baker, Stuart N. Isaacs, M. Shannon Keckler, Grace Kubin, Alison C. Mawle, Michael M. McNeil, Sharon Medcalf, Michael Merchlinsky, Howard L. Minkoff, Jay R. Montgomery, Richard W. Moyer, Lynda Osadebe, Larry K. Pickering, Greg A. Poland, James M. Schmitt, Eric M. Sergienko, Jean C. Smith, Neil M. Vora, Sixun Yang.

\footnotetext{
${ }^{1}$ National Center for Emerging and Zoonotic Infectious Diseases, CDC ${ }^{2}$ Emory University Rollins School of Public Health, Atlanta, Georgia; ${ }^{3}$ Advisory Committee on Immunization Practices; ${ }^{4}$ Infectious Diseases Epidemiology Research Unit, University of Pittsburgh, Pennsylvania.
}

\section{References}

1. Rotz LD, Dotson DA, Damon IK, Becher JA; Advisory Committee on Immunization Practices. Vaccinia (smallpox) vaccine: recommendations of the Advisory Committee on Immunization Practices (ACIP), 2001. MMWR Recomm Rep 2001;50(No. RR-10).

2. CDC. Newly licensed smallpox vaccine to replace old smallpox vaccine. MMWR Morb Mortal Wkly Rep 2008;57:207-8. 
3. Fenner F, Henderson D, Arita I, Jezek Z, Ladnyi I. Smallpox and its eradication. Geneva, Switzerland: World Health Organization; 1988.

4. Greenberg RN, Kennedy JS. ACAM2000: a newly licensed cell culturebased live vaccinia smallpox vaccine. Expert Opin Investig Drugs 2008;17:555-64. http://dx.doi.org/10.1517/13543784.17.4.555

5. Frey SE, Newman FK, Kennedy JS, et al. Comparison of the safety and immunogenicity of ACAM1000, ACAM2000 and Dryvax in healthy vaccinia-naive adults. Vaccine 2009;27:1637-44. http://dx.doi. org/10.1016/j.vaccine.2008.11.079

6. Sanofi-Pasteur. ACAM2000, (smallpox (vaccinia) vaccine, live) [package insert]. Swiftwater, PA: Sanofi-Pasteur; 2015. http://www.fda.gov/ downloads/BiologicsBloodVaccines/Vaccines/ApprovedProducts/ UCM142572.pdf

7. Verardi PH, Titong A, Hagen CJ. A vaccinia virus renaissance: new vaccine and immunotherapeutic uses after smallpox eradication. Hum Vaccin Immunother 2012;8:961-70. http://dx.doi.org/10.4161/hv.21080

8. MacNeil A, Reynolds MG, Damon IK. Risks associated with vaccinia virus in the laboratory. Virology 2009;385:1-4. http://dx.doi. org/10.1016/j.virol.2008.11.045

9. Paoletti E, Taylor J, Meignier B, Meric C, Tartaglia J. Highly attenuated poxvirus vectors: NYVAC, ALVAC and TROVAC. Dev Biol Stand 1995;84:159-63.

10. Moss B. Replicating and host-restricted non-replicating vaccinia virus vectors for vaccine development. Dev Biol Stand 1994;82:55-63.

11. Ahmed F, Temte JL, Campos-Outcalt D, Schünemann HJ; ACIP Evidence Based Recommendations Work Group (EBRWG). Methods for developing evidence-based recommendations by the Advisory Committee on Immunization Practices (ACIP) of the US Centers for Disease Control and Prevention (CDC). Vaccine 2011;29:9171-6. http://dx.doi.org/10.1016/j.vaccine.2011.08.005

12. Ahmed F. US Advisory Committee on Immunization Practices handbook for developing evidence-based recommendations [Version 1.2]. Atlanta, GA: CDC; 2013. http://www.cdc.gov/vaccines/acip/recs/GRADE/ about-grade.html
13. CDC. GRADE evidence tables—recommendations in MMWR. Atlanta, GA: US Department of Health and Human Services, CDC; 2015. http:// www.cdc.gov/vaccines/acip/recs/GRADE/table-refs.html

14. Poland GA, Grabenstein JD, NeffJM. The US smallpox vaccination program: a review of a large modern era smallpox vaccination implementation program. Vaccine 2005;23:2078-81. http://dx.doi.org/10.1016/j.vaccine.2005.01.012

15. Lane JM, Ruben FL, Neff JM, Millar JD. Complications of smallpox vaccination, 1968. N Engl J Med 1969;281:1201-8. http://dx.doi. org/10.1056/NEJM196911272812201

16. Lane JM, Ruben FL, Neff JM, Millar JD. Complications of smallpox vaccination, 1968: results of ten statewide surveys. J Infect Dis 1970;122:303-9. http://dx.doi.org/10.1093/infdis/122.4.303

17. Chosewood LC, Wilson DE; CDC; National Institutes of Health. Biosafety in microbiological and biomedical laboratories. 5th ed. Washington, DC: US Department of Health and Human Services, Public Health Service, CDC, National Institutes of Health; 2009.

18. Pirwitz S. HICPAC guidelines for isolation precautions. Am J Infect Control 1997;25:287-8. http://dx.doi.org/10.1016/S0196-6553(97)90017-1

19. Sehulster L, Chinn RY. Guidelines for environmental infection control in health-care facilities. Recommendations of CDC and the Healthcare Infection Control Practices Advisory Committee (HICPAC). MMWR Recomm Rep 2003;52(No. RR-10).

20. US Food and Drug Administration. ACAM2000 smallpox vaccine: Vaccines and Related Biological Products Advisory Committee (VRBPAC) briefing document. Washington, DC: US Food and Drug Administration; 2007. http://www.fda.gov/ohrms/dockets/ac/07/ briefing/2007-4292b2-02.pdf

21. Arness MK, Eckart RE, Love SS, et al. Myopericarditis following smallpox vaccination. Am J Epidemiol 2004;160:642-51. http://dx.doi. org/10.1093/aje/kwh269 